\title{
Method of Spatial Path Planning for Mobile ROBOT IN UNKNOWN ENVIRONMENT
}

\author{
Dmitry Yukhimets ${ }^{1,2}$, Alexander Zuev ${ }^{1,2} \&$ Anton Gubankov $^{1,2}$ \\ ${ }^{1}$ Institute of Automation and Control Processes FEB RAS, Vladivostok, Russia \\ ${ }^{2}$ Far Eastern Federal University, Vladivostok, Russia
}
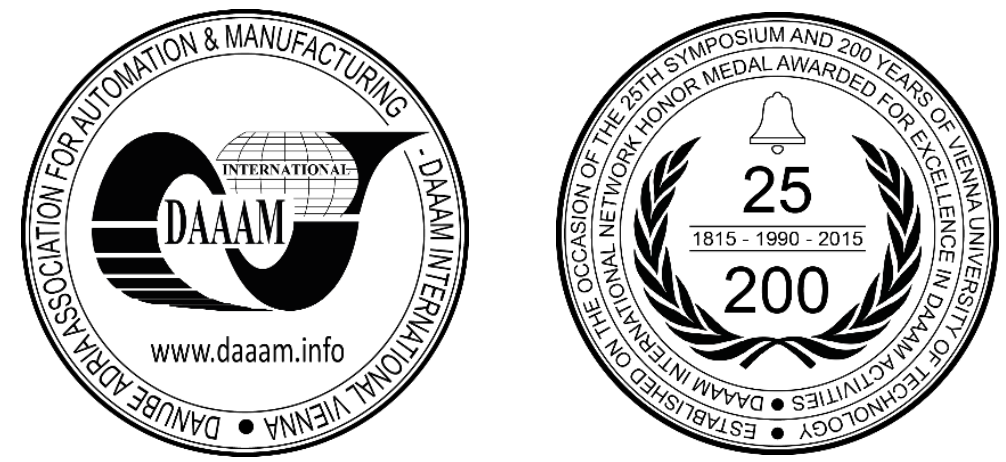

This Publication has to be referred as: Yukhimets, D[mitry]; Zuev, A[lexander] \& Gubankov, A[nton] (2017). Method of Spatial Path Planning for Mobile Robot in Unknown Environment, Proceedings of the 28th DAAAM International Symposium, pp.0258-0267, B. Katalinic (Ed.), Published by DAAAM International, ISBN 978-3-902734-11-2, ISSN 1726-9679, Vienna, Austria

DOI: $10.2507 / 28$ th.daaam.proceedings.035

\begin{abstract}
This paper deals with developing of method of spatial path planning for mobile robots of different types moving in unknown environment with obstacles. This method plans trajectories base on third-order Bezier splines using the data from onboard range sensors. It allows to automatically make decisions about the strategy of obstacles avoidance in horizontal or vertical planes considering the restrictions of mobile robots' propulsion system. Results of simulation confirm its high efficiency, low complexity and small computational capacity.
\end{abstract}

Keywords: mobile robot; path planning; obstacle avoidance; spline Bezier; smooth trajectory; unknown environment

\section{Introduction}

Today the application field of mobile robots (MR) of various types is continuously expanding. At the same time the tasks solved by the MR in the autonomous mode without control of an operator in conditions of an unknown in advance environment get special significance $[1,2]$. These tasks include transportation of cargos of various function, inspection of structures, search operations and so on. In the process of performance of these tasks the information control system (ICS) of the MR must form movement trajectories on the basis of information obtaining from onboard sensors. These trajectories allow the robot to move quickly and safely in an unknown environment containing obstacles.

The formation task of the MR trajectories in an environment containing obstacles is one of the main problems in development of the ICS for MR. Many methods which can be divided into two groups is already developed for it solution. The first group methods include the methods forming trajectories based on use of various methods of finding the shortest path. Such methods include algorithms of finding an optimal path on a graph [3,4], genetic algorithms [5-7] and other methods [8-10], which are used in the presence of a priori information describing obstacles location. In the case when the MR moves in a partially unknown or dynamic environment the trajectory should be planned dynamically based on 
information coming from the onboard MR sensors and predefined movement patterns [11, 12]. The disadvantage of the approaches of this group of methods is the high computational complexity and a need to develop special heuristic rules for the trajectories formation. In addition, the trajectories formed by them are a sequence of base points, over which the MR must pass. It requires additional application of various interpolation algorithms to construct smoothed motion trajectories.

The second group includes approaches based on the method of potential fields [13-14], which allow the MR to move in an unknown in advance environment. The methods form control signals applied to MR actuators as a sum of the robot reactions to the signals obtaining from their sensors. However, such approaches do not allow to control the robots' trajectories during their movement. Also, they can lead to a sharp change in the movement direction of the MR, which are poorly carried out by feedback systems of the robots.

Despite the large number of methods of the MR trajectories formation almost all of them consider the problem of plane motion. It significantly reduces the functionality of the MR moving in space (underwater vehicles (UV) and unmanned aerial vehicles (UAV)). The possibility of spatial motion allows these robots in some cases to avoid obstacles from above without changing the movement direction in the horizontal plane. Also, the orientation of its onboard sensors continuously changes, when the MR is moving in the vertical plane. It can distort the obstacles picture around the MR and leads to the design of incorrect motion trajectories, when traditional methods are used.

In some papers the methods of trajectories formation for the MR moving in space are proposed. In [15] the hybrid method for trajectory formation of a UAV in a mountainous region is described. In this method the optimal flat motion trajectory is first formed based on a mesh description of the UAV working space. The resulting discrete trajectory is smoothed using the method of potential fields. The main disadvantage of the described method is needing to know in advance the map of the location of all obstacles, as well as the inability to fly over the encountered obstacles. In [16] the methods of obstacles avoiding in the vertical plane based on the use of information from their onboard sensors are described. However, in these papers it is assumed that the UV has no restrictions on the pitch angle and can always bypass the obstacle from above. This assumption is valid only for certain UV having vertical thrusters.

So, there is the task of developing a method of formation of spatial trajectories of the MR spatial motion with unknown obstacles, based on information obtaining from onboard sensors. This method will allow to use all the possibilities of spatial movement of the MR. In the article this problem is solved on the basis of the formation method of smooth motion trajectories in an unknown environment [17]. This method will be modified taking into account the spatial character of the MR motion.

\section{Problem Statement}

When developing the path planning method for MR we assume that their trajectories are set in advance. These trajectories pass through the sequence $\mathbf{W}=\left\{W_{1}, W_{2}, \ldots, W_{n}\right\}$ of base points (where $W_{i}=\left(x_{W i}, y_{W i}\right)^{\mathrm{T}}$ ) and described by third order Bezier splines. Since the surface geometry is unknown in advance, the original trajectory of the MR is defined in a horizontal plane. Therefore, the $z$ coordinate for each point of the source trajectory will be formed in the process of robot movement so that he was always at a specified height from the surface.

To get the information about surrounding objects the MR have a set of onboard sensors, which generate signals about the distance from the robot to objects in the direction of the respective sensors. These sensors include different sonars and Doppler velocity logs (for underwater robots), 3D sonar, and various distance sensors (laser or ultrasonic). These sensors are divided into two groups: sensors that measure distances to obstacles located around MR, and sensors measuring the distance to the surface. Based on information received from these sensors is continuously generated two vectors $\mathbf{D}=\left(d_{1}, d_{2}, \ldots d_{i}, \ldots, d_{m}\right)$ and $\mathbf{H}=\left(h_{1}, h_{2}, \ldots h_{j}, \ldots, h_{n}\right)$, where $m$ and $n$ is the number of relevant onboard sensors.

Further, we assume that MR has four sensors of distance to the surface, directed forward, backward, right and left relative to MR. (see Fig. 1). This situation is standard Doppler logs used onboard of UV.

Let's suppose that data obtained from onboard sensors satisfy the following conditions.

1. The zero-signal generated at the output of the $i$-th sensor indicates that the obstacle in the corresponding direction is not detected.

2. The range of detection of obstacles is limited by the value $d_{\max }$, which can be different for different groups of sensors, but for simplicity is the same for all sensors.

3. Time of filling of vectors $\mathbf{D}$ and $\mathbf{H}$ by the data obtained from multiple independent onboard distance sensors does not exceed the sampling period ICS of the robot.

4. The orientation of all the sensors in the coordinate system of the MR is known and does not change during robot movement. In the article it is supposed that all sensors in each group are arranged symmetrically about the roll axis of MR. 


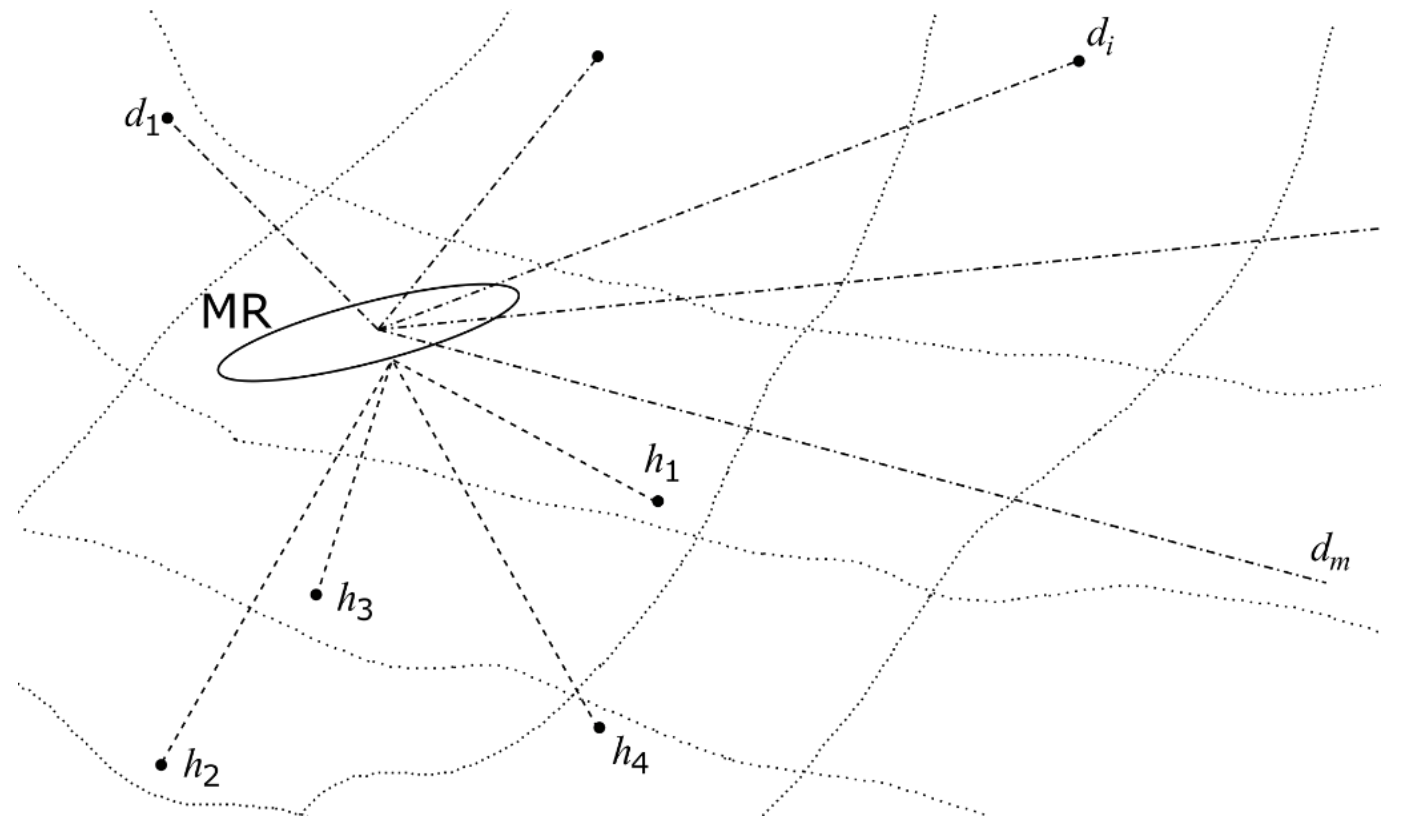

Fig. 1. Signal from onboard range sensors

Since the movement of the robot takes place in an unknown environment, the trajectory of this movement is defined in advance using a set of reference points. These points need to be adjusted in the process of movement based on data continuously received from onboard MP sensors. Thus, the process of generating of spatial trajectories have the following requirements.

1. The correction of these trajectories in the process of robot movement should not lead to an abrupt change of direction and speed of this movement.

2. Generated trajectory should allow the MR to going through the all base points in the specified sequence.

3. The generated trajectory should pass not less than the allowable distance $D_{\min }$ from the detected obstacle which is determined by the dynamic characteristics and the MR type.

4. All the parts of generated trajectories should be at a given height $H_{d}$ from the surface of the robot movement.

5. The movement along the generated trajectories should not lead to the formation of the MR pitch modulo greater than the maximum allowed value $\varphi_{\max }$. This requirement is due to the features of construction and propulsion complexes of various types of MR.

As a basic method of forming the trajectory of the MR the method described in [17] will be used. This method generates and continuously tunes the smooth trajectory of the MR described by Bezier splines in the horizontal plane according to the data obtained from MR onboard sensors. This ensure the safe movement of the robot in a previously unknown environment containing obstacles. To use this method for MR performing spatial motion it is necessary to make the ability to avoid obstacles in the vertical plane and the always move at a given height from a surface with an unknown in advance relief.

Thus, the article solves the following problem. Let's set the initial smooth, flat trajectory of the robot movement, passing through a sequence of base points W. MR is moving in a previously unknown environment and equipped with sensors forming a vector $\mathbf{D}$ of distances to the detected obstacles and the vector $\mathbf{H}$ of the distances to the surface over which the movement of the robot is performing. It is necessary to correct the original trajectory that the resulting trajectory passes through the entire sequence of base points $\mathbf{W}$, was located at the height $H_{d}$ of the surface of the movement and the distance to the detected obstacles should not be smaller than the allowable $D_{\text {min }}$.

The task of correcting the trajectory of the MR is solved in several steps.

Step 1. Analysis of environment based on data received from onboard MR sensors.

At this step there is evaluation of the surface relief and calculation of the coordinates of reference point $X_{d}$ that specifies the movement of the robot. The specified coordinates $X_{d}$ are calculated based on the current trajectory in the horizontal plane, the surface relief and the given speed of robot movement along the trajectory.

Step 2. Analysis of the location of obstacles relative to the MR and selecting of strategy of obstacle avoidance.

Based on information obtained from onboard sensors the MR evaluates magnitude of the pitch angle sufficient to avoid obstacles in the vertical plane. If this value is less than $\varphi_{\max }$, then MR can go around the obstacle in a vertical plane without correction of the trajectory in the horizontal plane. Otherwise, it makes decision of correction of the trajectory in the horizontal plane and avoid obstacles on the side saving the given height above the surface.

Step 3. The calculation of the parameters of the new trajectory using the algorithm described in [17], if MR makes the decision of correction.

Further, all these steps are described more detail. 


\section{Algorithm of forming of spatial trajectories of MR movement}

Before beginning the work of the algorithm of formation of spatial trajectories ICS calculates the coordinates of points lying on obstacles and on surface of movement determined by the distance sensors. These coordinates considering the spatial orientation of MR is calculated as follows:

1) for sensors $d_{i}$ :

$$
X_{d i}=X+R_{G}(\theta, \varphi, \psi) R_{M R}\left(a_{d i}, b_{d i}, c_{d i}\right)\left[\begin{array}{c}
0 \\
0 \\
d_{i}
\end{array}\right], i=(\overline{1, m}),
$$

where $X=(x, y, z)$ are coordinates of MR in world coordinate system; $\theta, \varphi, \psi$ are roll, pitch and yaw of MR respectively; $a_{d i}, b_{d i}, c_{d i}$ are angles of orientation of $i$-th sensor about the axis $x, y$ and $z$ in the MR body-fixed coordinate system; $R_{G}($.$) ,$ $R_{M R}($.) are the rotation matrixes along axis $x, y$ and $z$ in world and body-fixed coordinate systems respectively;

2) for sensors $h_{j}$ :

$$
X_{h j}=X+R_{G}(\theta, \varphi, \psi) R_{M R}\left(a_{h j}, b_{h j}, c_{h j}\right)\left[\begin{array}{c}
0 \\
0 \\
h_{j}
\end{array}\right], j=(\overline{1,4}),
$$

where $a_{h j}, b_{h j}, c_{h j}$ are orientation angles of $j$-th surface distant sensor in in the MR body-fixed coordinate system. For definiteness, we assume that the point $X_{h 1}$ is ahead of MR, point $X_{h 2}$ is behind MR, point $X_{h 3}$ is on the left and point $X_{h 4}$ is on the right of the MR (see. fig. 1).

After determining the coordinates $X_{h j}$ the coordinates of reference point $X_{d}$ are calculated. They specify the desired movement of the robot along the trajectory. This calculation performs in two steps. At the first step the value of desired pitch angle $\varphi_{d}$ is calculated. With $\varphi_{d}$ the MR should move along the path to its height above the surface was equal to the given value $H_{d}$. On the second step the coordinates of the point $X_{d}$ are calculated considering given trajectory in the horizontal plane, the $\varphi_{d}$ and the value of $v_{d}$, which is reference speed of MR movement.

To calculate the $\varphi_{d}$ the coordinates $X_{h 1}$ and $X_{h 2}$ are used. In the calculation it is considered that the profile of the surface relief under MR can be approximated by a straight line passing through these points (see fig. 2).

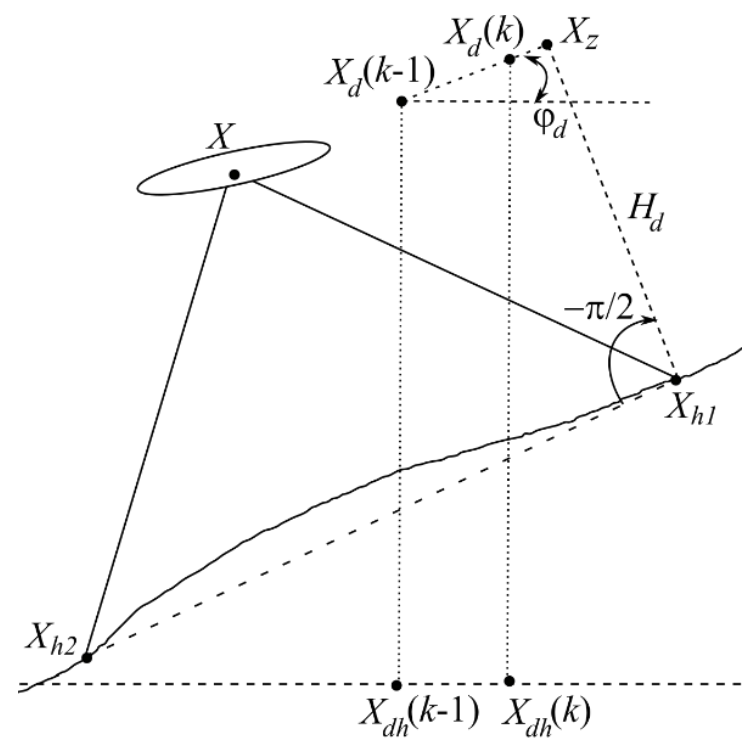

Fig. 2. Coordinate calculation of reference point $X_{d}$

In the beginning the coordinates of point $X_{z}$ is calculated. This point is situated on distance $H_{d}$ from point $X_{h 1}$ and on the plane passed through the points $X, X_{h 1}$ и $X_{h 2}$ (Fig.2). These coordinates are calculated by following expressions:

$$
X_{z}=R_{\psi \theta \psi} \frac{X_{h 1}-X_{h 2}}{\left\|X_{h 1}-X_{h 2}\right\|} H_{d}+X_{h 1},
$$


where $R_{\psi \theta \psi}=R_{G}(0,0, \psi) R_{G}(0,-\pi / 2,0) R_{G}(0,0,-\psi)=\left[\begin{array}{ccc}\sin ^{2} \psi & -\cos \psi \sin \psi & -\cos \psi \\ -\cos \psi \sin \psi & \cos ^{2} \psi & -\sin \psi \\ \cos \psi & \sin \psi & 0\end{array}\right]$. The matrix $R_{\psi \theta \psi}$ defines

the rotation of vector $\left(X_{h 1}-X_{h 2}\right)$ on angle $\pi / 2$ relatively axis perpendicular this vector and lying in horizontal plan. If we know of point $X_{z}$ coordinates we can calculate of value $\varphi_{d}$ by following expression:

$$
\varphi_{d}=\arctan \left(\frac{z_{z}-z_{d}(k-1)}{\left\|\left[x_{z}, y_{z}\right]-\left[x_{d}(k-1), y_{d}(k-1)\right]\right\|}\right),
$$

where $\left(x_{z}, y_{z}, z_{z}\right)$ и $\left(x_{d}(k-1), y_{d}(k-1), z_{d}(k-1)\right)$ are the coordinates of points $X_{z}$ and $X_{d}(k-1)$, respectively. Index $k-1$ means coordinates $X_{d}$, calculated on previous sampling interval of MR control system.

Further we calculate the coordinates of point $X_{d}(k)=\left(x_{d}(k), y_{d}(k), z_{d}(k)\right)$ with considering of value $\varphi_{d}$ by following expressions:

$$
\begin{aligned}
& X_{d h}(k)=(1-\delta(k))^{3} W_{1}+3 \delta(k)(1-\delta(k))^{2} P_{1}+3 \delta(k)^{2}(1-\delta(k)) P_{2}+\delta(k)^{3} W_{2}=f_{x}(\delta(k)) \\
& z_{d}(k)=z_{d}(k-1)+\tan \left(\varphi_{d}\right)\left\|X_{d h}(k)-X_{d h}(k-1)\right\|
\end{aligned}
$$

where $W_{1}=\left(x_{W 1}, y_{W 1}\right), W_{2}=\left(x_{W 2}, x_{W 2}\right), P_{1}=\left(x_{P 1}, y_{P 1}\right), P_{2}=\left(x_{P 2}, x_{P 2}\right)$ are coordinates of starting, finishing and control points of spline describing current part of MR trajectory in horizontal plane; $X_{d h}=\left(x_{d}, y_{d}\right)$ is projection of point $X_{d}$ on horizontal plane; $\delta=[0,1]$ is parameter set the point $X_{d h}$ movement along spline from point $W_{1}$ to point $W_{2}$. The indexes k and k-1 mean values of different variables calculated for current and previous sampling interval of MR control system.

As we can see from expressions (5) the point $X_{d}$ coordinates is defined by $\delta(k)$. Thus for calculation of $X_{d}$ coordinates we have to find such value $\delta(k)$, which provide movement of point $X_{d}$ with reference speed $v_{d}$. This task is solved by following iterative algorithm.

Step 1. $S_{d}=v_{d} \Delta \tau, p=0, \delta_{t}(p)=\delta(k-1), S(p)=0, X_{d t}(p)=X_{d h}(k-1)$,

where $S_{d}$ is the path of point $X_{d}$ in a time $\Delta \tau$ when it moves with speed $v_{d} ; \Delta \tau$ is the sampling interval of MR control system; $p$ is the index of current iteration of algorithm.

Step 2. General calculation step.

$p=p+1$,

$\delta_{t}(p)=\delta_{t}(p-1)+\Delta$,

$X_{d t}(p)=f_{x}\left(\delta_{t}(p)\right)$,

$S_{x}=\left\|X_{d t}(p)-X_{d t}(p-1)\right\|$,

$S_{z}=\mid \tan \left(\varphi_{d}\right) \| S_{x}$,

$S(p)=S(p-1)+\left(S_{x}^{2}+S_{z}^{2}\right)^{1 / 2}$,

where $\Delta$ is the small increment of $\delta_{t}$.

Step 3. Checking of condition for calculation finish.

If $S(p) \geq S_{d}$, then $\delta(k)=\delta_{t}(p)$, else goto step 2 .

The coordinates of point $X_{d}$ for current sampling interval are calculated by expressions (5) after defining of $\delta(k)$. For increasing of dynamic accuracy $v_{d}$ can be tuned according to $[18,19]$.

MR can meet obstacles during movement along trajectory ant it must avoid these obstacles on safely distance. For this purpose, it is necessary to define presence of obstacles near MR based on information about points $X_{d i}, i=(\overline{1, m})$ and $X_{h j}, j=(\overline{1, n})$ and select the method of avoidance: in vertical plan without correction of trajectory in horizontal plane or sidewise with correction of trajectory in horizontal plan by algorithm described in [17]. This selection is made by estimation of value $\varphi^{*}$ pitch angle needed for obstacle avoidance in vertical plane.

All range sensors $d_{i}$ for calculation of $\varphi^{*}$ are separated on three groups (Fig. 3): range sensors of MR left side $\mathbf{L}=$ $\left(d_{l}, \ldots, d_{l}\right)$, range sensors of MR forward side $\mathbf{F}=\left(d_{l+1}, \ldots, d_{l+f}\right)$ and MR right side $\mathbf{R}=\left(d_{l+f+1}, d_{l+f+r}\right)$, where $l+f+r=m$. Each these groups associate with one of range sensor $h_{3}, h_{l}, h_{2}$ (Fig. 3). For each pair sensors $d_{i}$ and $h_{j}$ the estimation of pitch angle is calculated:

$$
\varphi_{i}^{*}=\left\{\begin{array}{l}
\arctan \left(\frac{z_{d i}-z_{h j}}{\left\|\left[x_{d i}, y_{d i}\right]-\left[x_{h j}, y_{h j}\right]\right\|}\right), \text { if } d_{i} \neq 0 \\
0, \text { if } d_{i}=0
\end{array}, i=\left\{\begin{array}{l}
(\overline{1, l}), \text { if } j=2 \\
(\overline{l+1, l+f}), \text { if } j=1 \\
(\overline{l+f+1, l+f+r}), \text { if } j=3
\end{array},\right.\right.
$$


where $x_{d i}, y_{d i}, z_{d i}$ are coordinates of point $X_{d i} ; x_{h j}, y_{h j}, z_{h j}$ are the coordinates of point $X_{h j}$.

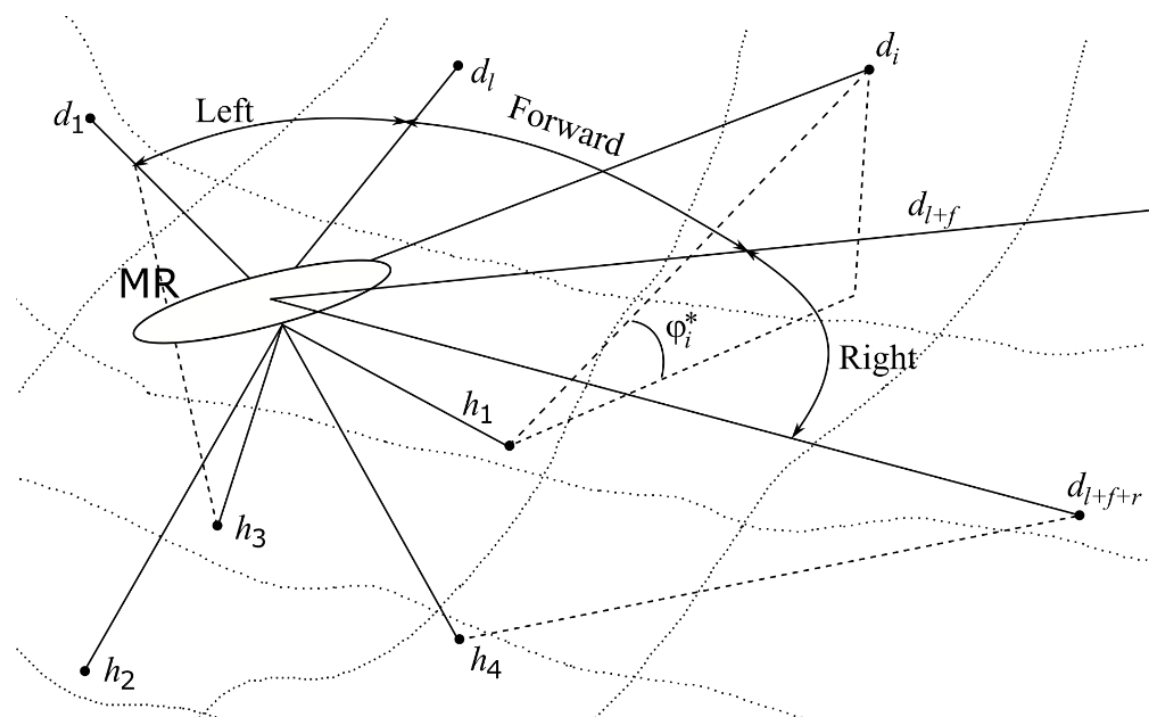

Fig 3. The selection of the method of obstacle avoidance

Finally, the value $\varphi^{*}$ is defined as $\varphi^{*}=\max \left(\varphi_{i}^{*}\right), i=(\overline{1, m})$. If $\varphi^{*} \leq \varphi^{*}$ max , then obstacle avoidance in vertical plan is possible and correction of MR trajectory in horizontal plane is not required. If $\varphi^{*}>\varphi^{*}$ max , then obstacle avoidance must be in horizontal plane with correction MR trajectory. Herewith the value $\varphi^{*}{ }_{\text {max }}$ is defined by following expression:

$$
\varphi_{\max }^{*}=\left\{\begin{array}{l}
\min \left(\arctan \left(\frac{H_{d}}{D_{\min }}\right), \varphi_{\max }\right), \text { if } d_{i} \in \mathbf{L} \text { or } d_{i} \in \mathbf{R} \\
\varphi_{\max }, \text { if } d_{i} \in \mathbf{F}
\end{array}, i=(\overline{1, m}) .\right.
$$

As we can see from (7) if obstacle is left or right side of MR then value $\varphi^{*}$ max depend on relation of values $H_{d}$ and $D_{\text {min }}$ and can be less then value $\varphi_{\max }$. The explanation of this fact is shown in Fig. 4.

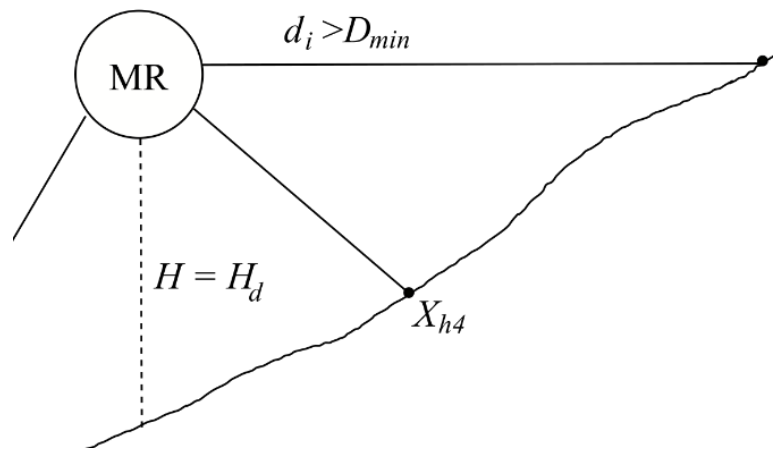

a)

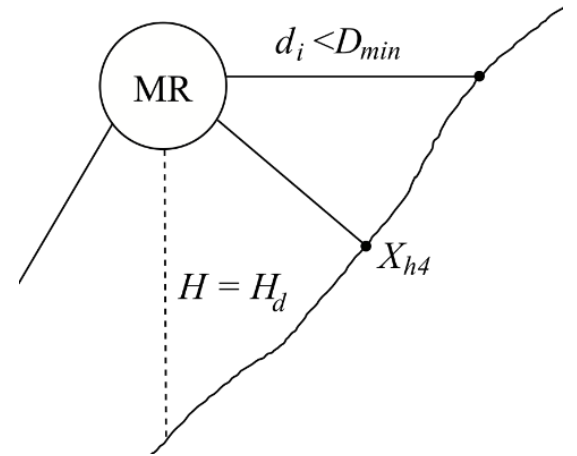

b)

Fig 4. The features of MR movement along different obstacles

The MR movement along obstacle with smooth slope being from side of MR is shown in the Fig. 4a. In this case the MR can keep reference altitude from surface and simultaneously keep distance from obstacle more than minimal value $D_{\text {min. }}$. But if MR move along obstacle with stiff slope (Fig. 4b) then it became impossible simultaneously keep reference altitude from surface and minimal allowed distance from obstacle. If MR shifts from obstacle for increasing of side distance then altitude decrease too and MR come down. As result the side distance decrease again. So, for simultaneously keeping and altitude and minimal allowed side distance the value $\varphi^{*}$ max must consider of relation between values $H_{d}$ and $D_{\text {min. }}$

If obstacle must be avoiding by sidewise then trajectory setting in horizontal plane by Bezier spline of third degree is corrected by algorithm proposed in [17]. Herewith depend on obstacles position relatively MR there are two methods of trajectory correction: obstacle avoidance (from left or right side) or shifting trajectory for providing safely distance from 
obstacle. After correction the current part of MR trajectory is described by spline with new parameters which are used in expressions (5) for calculation of coordinates of point $X_{d}$.

\section{The simulation result of proposed MR spatial path planning method}

The mathematical simulation was carried out for investigation of proposed method. During this simulation the V-rep software is used for building of virtual training area (Fig. 5) containing different obstacles and MATLAB software is used for implementation this method of path planning.

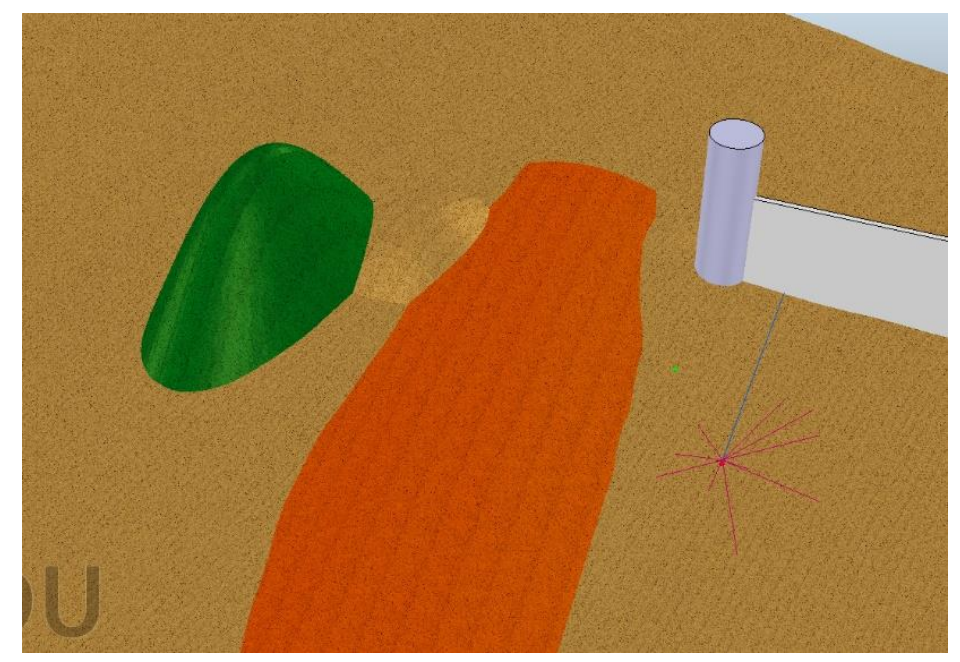

Fig. 5. General view of virtual training area

During simulation it is proposed that MR has 7 range sensors of distance to obstacles and 4 range sensors of distance to surface. Sensors of distance to surface are directed to forward, backward, left side and right side relatively MR and have declination from yaw axis on angles $\pi / 4 \mathrm{rad}$ in corresponding side. The reference altitude value was equal $H_{d}=2 \mathrm{~m}$, minimal allowed distance from obstacles was equal $D_{\min }=2 \mathrm{~m}$, maximal allowed value of pitch angle was equal $\varphi_{\max }=$ $0.6 \mathrm{rad}$ and reference MR velocity was equal $v_{d}=1 \mathrm{~m} / \mathrm{s}$. Starting point W1 has coordinates $(24,24)$, finishing point W2 has coordinates $(-60,30)$, and arrival angle to finishing point was equal $\pi$ rad. Starting value of $z$ coordinate was equal 4 $\mathrm{m}$. During simulation it is supposed that MR control system provide its accurate movement along trajectory [20] and yaw and pitch angles are formed by direction of tangent lines to this trajectory in horizontal and vertical planes.

The simulation results of MR movement are presented in Fig. 6. In Fig. 6a the gray dashed line is the initial trajectory set in horizontal plane, and black solid line is the projection of resulting MR trajectory to horizontal plane. In Fig. $6 \mathrm{~b}$ the changing of coordinate $\mathrm{z}$ along trajectory shown in Fig. 6a is presented. General view of resulting trajectory on virtual training area is shown in Fig. 6c. As we can see in these figures during MR movement its meet two obstacles: smooth and sharp hills. First obstacle MR avoids in vertical plan without correction of path in horizontal plane (gray and black line coincide in Fig. 6a). But MR cannot avoid second obstacle in vertical plane because of limitation of its pitch angle. In this case the path is corrected for providing avoidance of this obstacle from left side.

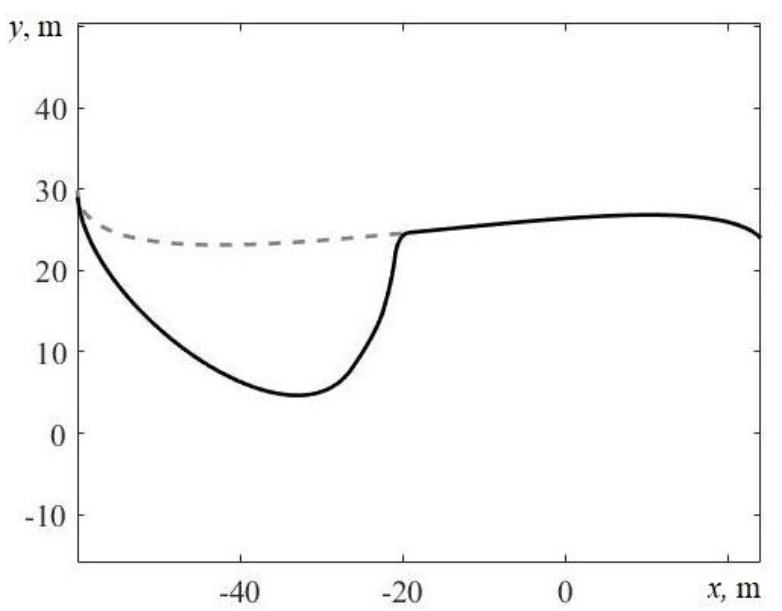

a)

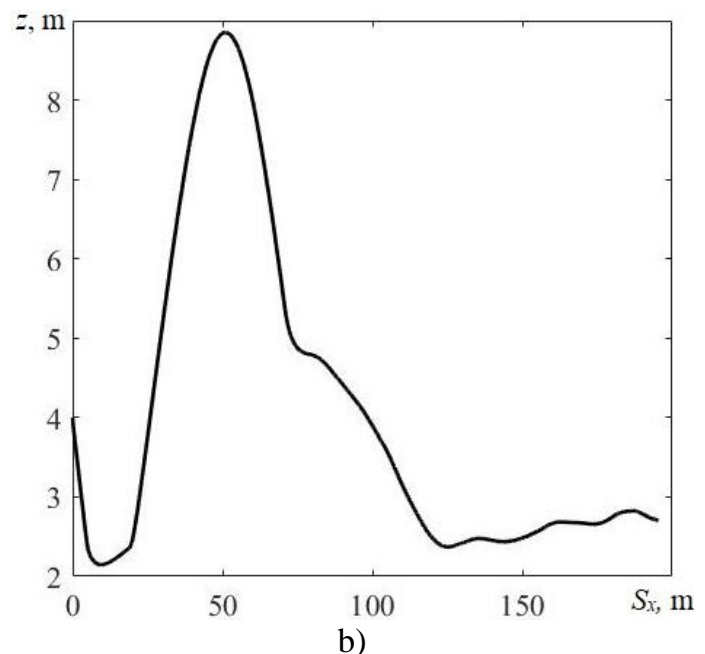

b) 


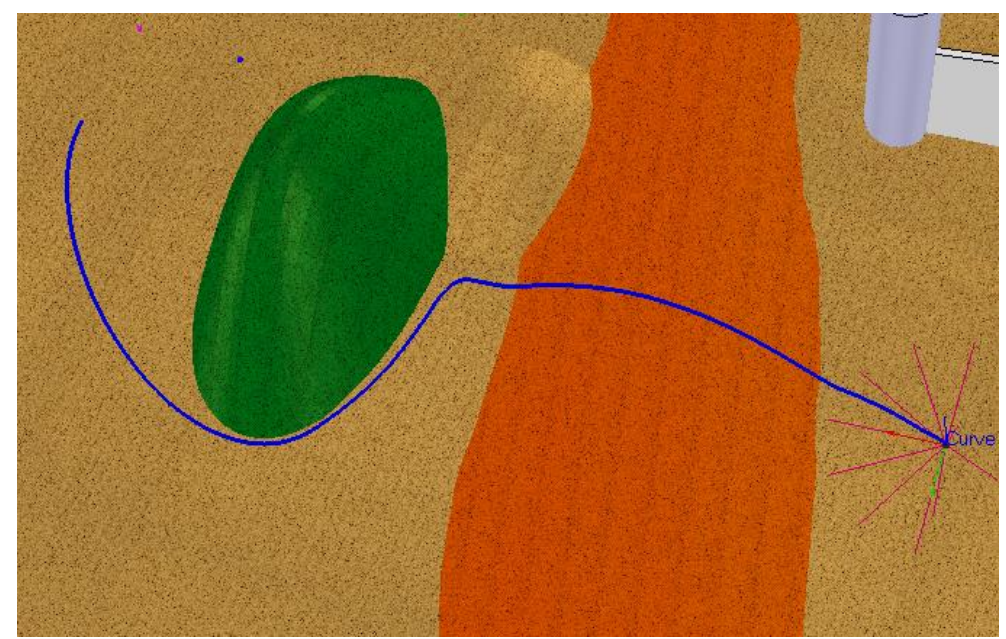

c)

Fig. 6. The resulting of MR trajectory

In the Fig. 7a the value $\varphi^{*}$ changing is shown. As we can see when MR avoids first obstacle in vertical plane the value $\varphi^{*}<\varphi^{*}$ max and when it avoids second obstacle $\varphi^{*}<\varphi^{*}{ }_{\max }$, and as result the initial trajectory is changed. In the Fig. 7b the changing of minimal distance from obstacles measured by range sensors is shown. In this figure zero distance means that there are no obstacles located near MR.
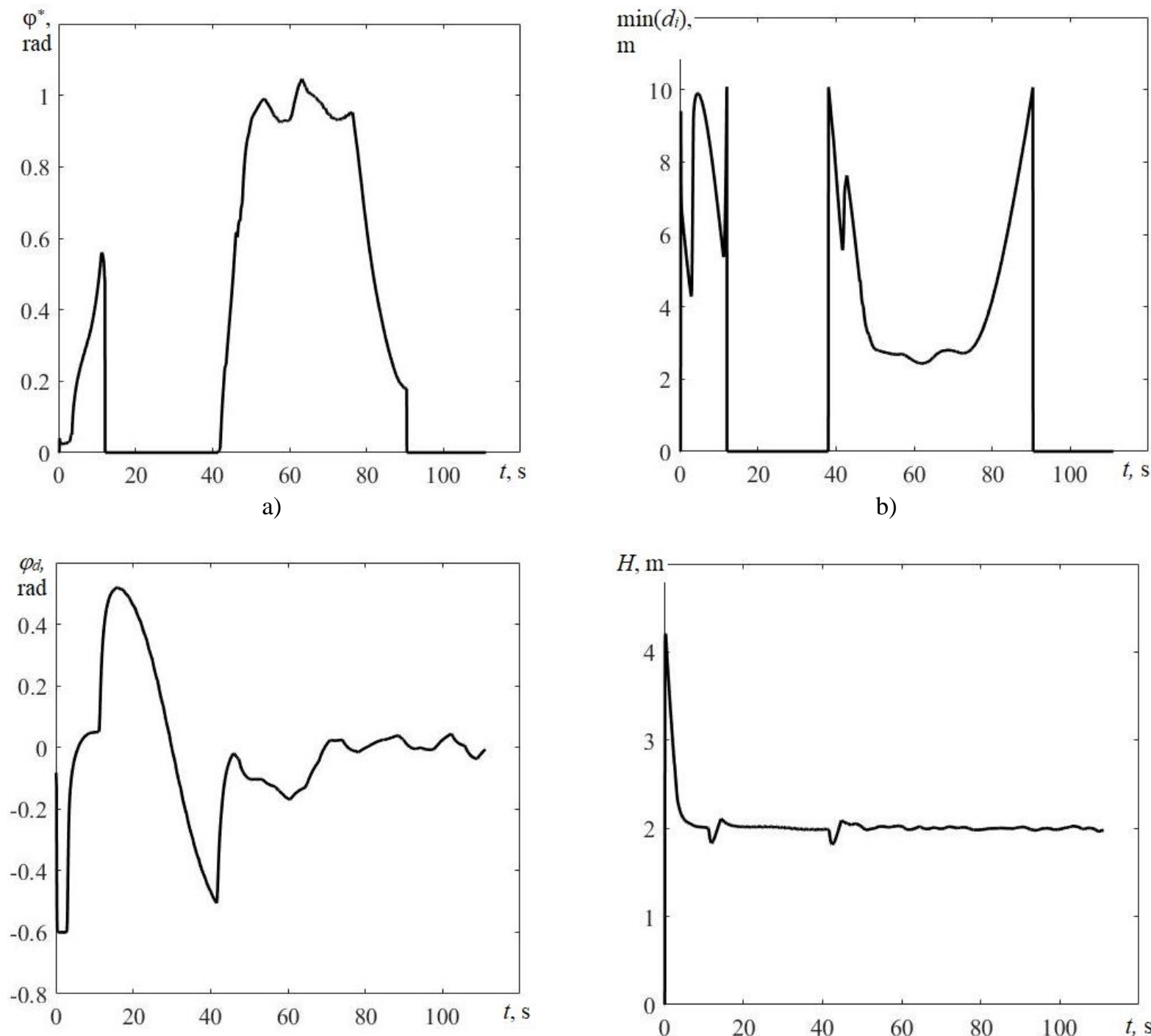

c)

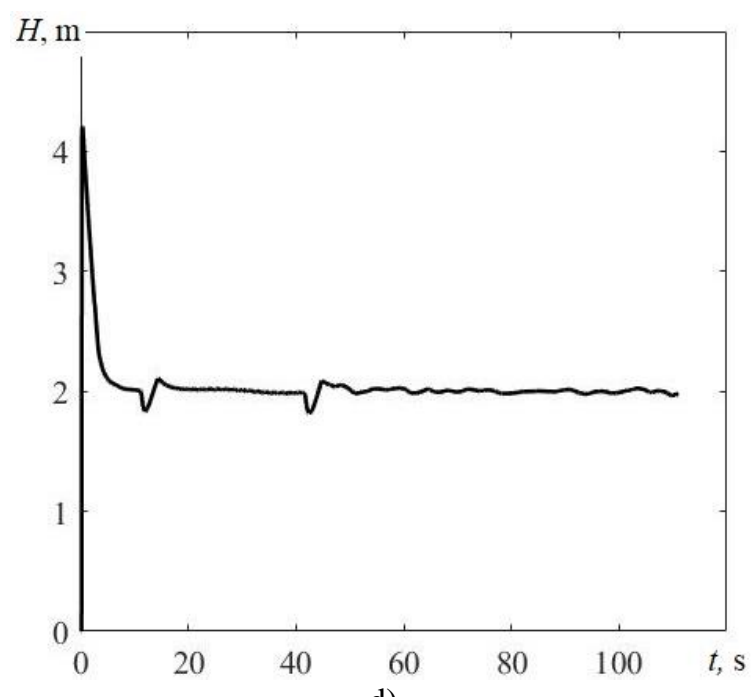

d) 


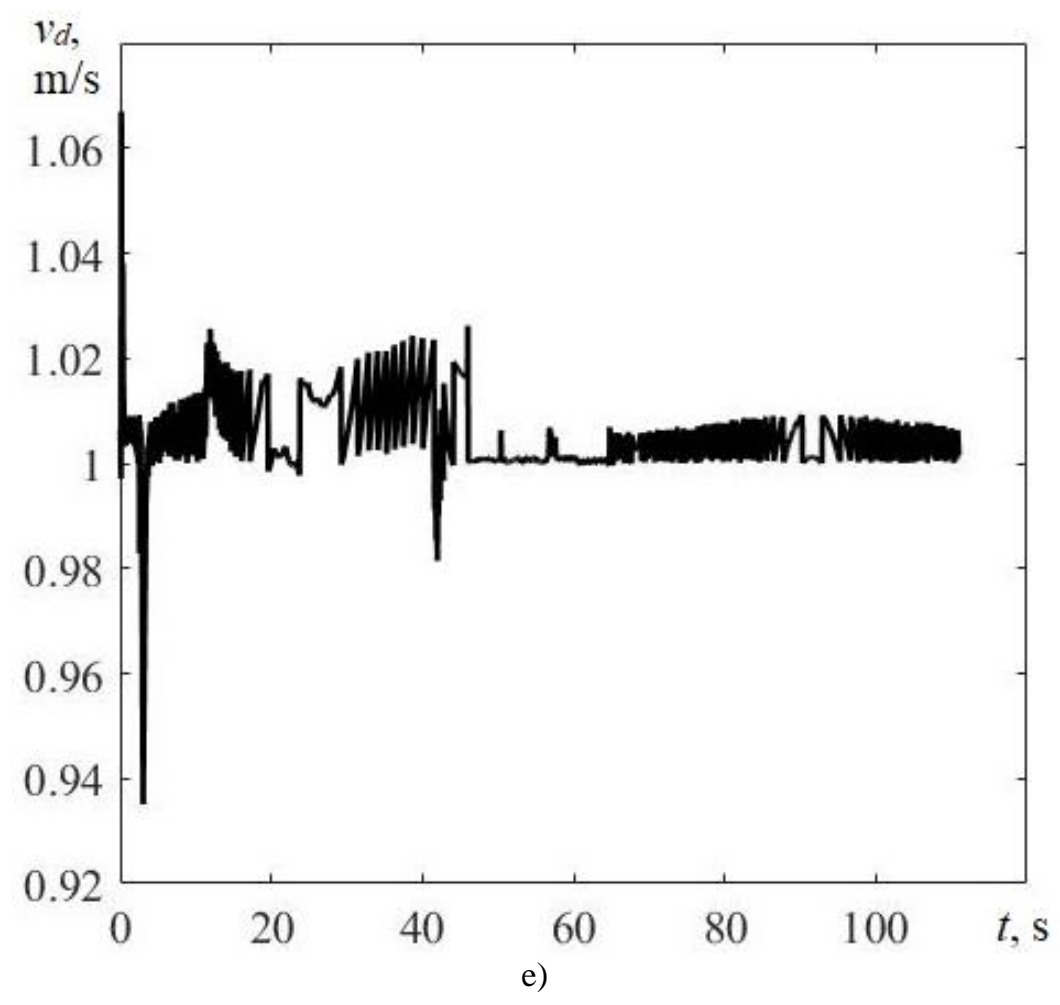

Fig. 7. Changing $\varphi^{*}(\mathrm{a}), \min \left(d_{i}\right), i=(1, m),(\mathrm{b}), \varphi_{d}(\mathrm{c}), H(\mathrm{~d}), v_{d}(\mathrm{e})$ during MR movement

As we can see in this figure during MR movement minimal distance from obstacles always more than minimal allowed distance. Herewith this distance is minimal when MR moves along second obstacle. During movement the value of MR pitch angle not exceed $\varphi_{\max }=0.6 \mathrm{rad}$ (Fig. 7c). and deviation of altitude $H$ under surface from reference value of altitude does not exceed $0.15 \mathrm{~m}$ (Fig. 7d). This deviation has maximal value when MR starts and finishes avoidance of first obstacle in vertical plane where relief of surface changes sharp. In other time this deviation does not exceed $0.07 \mathrm{~m}$. The changing velocity of point $X_{d}$ is presented in Fig. 7e. As we can see the deviation this velocity from its reference value does not exceed $0.02 \mathrm{~m} / \mathrm{s}$ that provides performing any operations.

Thus, the simulation results confirm that proposed algorithm to allow planning spatial trajectories for MR in unknown environment and simultaneous keeping of reference altitude from surface and avoiding obstacles on safe distance. Also proposed algorithm has small computation complexity and allow automatically select mode of obstacle avoidance.

\section{Conclusions}

This paper proposes the new method of formation of spatial trajectories of the robot movement in an unknown environment containing obstacles, based on data obtained from onboard distance sensors. The proposed method is based on the previously developed method of formation of smooth trajectories. Feature of the proposed method is the simple mechanism of making decisions about bypass mode for detected obstacles: in the vertical plane without correction for the original trajectory, or side with the correction of this trajectory. The advantages of the proposed algorithm can indicate the formation of smooth trajectories, which allows to increase the speed of robot movement and its small computational complexity.

The future work will be continued according two ways. The first way is developing of intelligent approach to select the mode of obstacle avoidance in horizontal or vertical plans. For instance, on the base of fuzzy logic methods. The second way is developing method of selecting direction of obstacle avoidance on the base of analysis of covered distance.

\section{Acknowledgments}

This work is supported by RFBR (16-37-60025, 16-29-04195) and grant of President of Russia (MK-8536.2016.8).

\section{References}

[1] Fossen, T.I. (2011). Handbook of Marine Craft Hydrodynamics and Motion Control. Chichester: Wiley\&Sons Ltd, ISBN: 978-1-119-99149-6

[2] Siciliano B. \& Khatib O. Handbook of Robotics. (2008). Heidelberg: Springer-Verlag, Berlin. 
[3] Wangy L.; Yongy L. \& Marcelo H. (2002). Hybrid of global path planning and local navigation implemented on a mobile robot in indoor environment, Proceedings of the 2002 IEEE International Symposium on Intelligent Control (ISIC). Vancouver, British Columbia, Canada. pp. 821-826.

[4] Wu Zh. \& Feng L. (2012). Obstacle prediction-based dynamic path planning for a mobile robot, International Journal of Advancements in Computing Technology, Vol. 4, No. 3, pp. 118-124.

[5] Jianping T. \& Yang S. (2003). Genetic algorithm based path planning for a mobile robot, Proc. of the IEEE International Conference on Robotics and Automation, Taipei, Taiwan, Vol. 1, pp. 1221-1226.

[6] Shiltagh N. \& Jalal L. (2013). Path Planning of Intelligent Mobile Robot Using Modified Genetic Algorithm, International Journal of Soft Computing and Engineering (IJSCE), Vol. 3, No. 2, pp. 31-36.

[7] Sedighi K.H.; Ashenayi K.; Manikas T.W.; Wainwright, R.L. \& other. (2004). Autonomous Local Path-Planning for a Mobile Robot Using a Genetic Algorithm, Proc. of the Congress on Evolutionary Computation. Portland, USA, Vol. 2, pp.1338-1345.

[8] Shiltagh, N. \& Jalal L. (2013). Optimal path planning for intelligent mobile robot navigation using modified particle swarm optimization, International Journal of Engineering and Advanced Technology, Vol. 2, No. 4, pp. $260-267$.

[9] Garrido S.; Moreno L.; Blanco D. \& Jurewicz P. (2011). Path planning for mobile robot navigation using Voronoi diagram and fast marching, International Journal of Robotics and Automation, Vol. 2, No. 1, pp. $42-64$.

[10] Mohanraj T.; Arunkumar S.; Raghunath M. \& Anand M. (2013). Mobile robot path planning using ant colony optimization, IJRET: International Journal of Research in Engineering and Technology, Vol. 213, No. 11, pp. 1-6.

[11] Saha O. \& Dasgupta P. (2017). Real-time robot path planning around complex obstacle patterns through learning and transferring options, Proc. of the 2017 IEEE International Conference on Autonomous Robot Systems and Competitions (ICARSC), pp. 278-283.

[12] Jiang M.; Chen Y.; Zheng W.; Wu H. \& Cheng L. (2017). Mobile robot path planning based on dynamic movement primitives, Proc. of the IEEE International Conference on Information and Automation, pp. 980-985.

[13] Ge S. \& Cui Y. (2002). Dynamic motion planning for mobile robots using potential field method, Autonomous Robots, Vol. 13, pp. 207-222.

[14] Zhou J.-H. \& Lin H.-Y. (2013). A self-localization and path planning technique for mobile robot navigation, Proceedings of the 8th World Congress on Intelligent Control and Automation, Taipei, Taiwan. pp. 694-699.

[15] Tan J.; Zhao L.; Wang Y.; Zhang Y. \& Li L. (2016). The 3D Path Planning Based on A* Algorithm and Artificial Potential Field for the Rotary-Wing Flying Robot, Proc. of the 8th International Conference on Intelligent HumanMachine Systems and Cybernetics, pp. 551-556.

[16] Ageev M.D. (ed.). (2005). Autonomous underwater robots: systems and technology, Nauka, Moskow.

[17] Filaretov V.F. \& Yukhimets D.A. (2017). Planning smooth paths for mobile robots in an unknown environment, Int. Journal of Computer and Systems Sciences, Vol. 56, No. 4, pp. 738-748.

[18] Filaretov V.F.; Gubankov A.S. \& Gornostaev I.V. (2016). The formation of motion laws for mechatronics objects along the paths with the desired speed, Proc. of International Conference on Computer, Control, Informatics and Its Applications (IC3INA 2016), 03 - 05 October 2016, Jakarta, Indonesia. pp. 93-96.

[19] Filaretov, V. F. \& Gubankov, A. S. (2012). Adaptive system forming extremely high speed of multilink manipulator gripper, Annals of DAAAM for 2012 \& Proceedings of the 23rd International DAAAM Symposium, ISBN 978-3901509-91-9, ISSN 2304-1382, pp. 0473 - 0476, B. Katalinic (Ed.), Published by DAAAM International, Vienna, Austria.

[20] Filaretov, V.F. (2007). Synthesis of Adaptive Control Systems for Electric Servo Actuators of Manipulators, Proceedings of the 28th DAAAM International Symposium, pp. 277- 279, B. Katalinic (Ed.), Published by DAAAM International, ISSN 1726-9679, ISBN 3-901509-58-5, Zadar, Croatia. 